\title{
BMJ
}

\section{Continuous deep sedation for patients nearing death in the Netherlands: descriptive study}

\author{
Judith Rietjens, postdoctoral researcher, ${ }^{1}$ Johannes van Delden, professor of medical ethics, ${ }^{2}$ \\ Bregje Onwuteaka-Philipsen, associate professor, ${ }^{3}$ Hilde Buiting, junior researcher, ${ }^{1}$ Paul van der Maas, \\ professor of public health, ${ }^{1}$ Agnes van der Heide, associate professor ${ }^{1}$
}

${ }^{1}$ Department of Public Health, Erasmus MC, PO Box 2040, 3000 CA Rotterdam, Netherlands 2Julius Centre for Health Sciences and Primary Care, University Medical Centre Utrecht,

PO Box 85500, 3508 GA Utrecht, Netherlands

${ }^{3}$ Department of Public and Occupational Health, Institute for Research in Extramural Medicine VU University Medical Centre, 1081 BT Amsterdam, Netherlands Correspondence to: J Rietjens j.rietjens@erasmusmc.nl

doi:10.1136/bmj.39504.531505.25

\section{ABSTRACT}

Objectives To study the practice of continuous deep sedation in 2005 in the Netherlands and compare it with findings from 2001.

Design Questionnaire study about random samples of deaths reported to a central death registry in 2005 and 2001.

Setting Nationwide physician study in the Netherlands. Participants Reporting physicians received a questionnaire about the medical decisions that preceded the patient's death; 78\% $(n=6860)$ responded in 2005 and $74 \%(n=5617)$ in 2001.

Main outcome measures Characteristics of continuous deep sedation (attending physician, types of patients, drugs used, duration, estimated effect on shortening life, palliative consultation). Requests for euthanasia.

Results The use of continuous deep sedation increased from $5.6 \%$ (95\% confidence interval $5.0 \%$ to $6.2 \%$ ) of deaths in 2001 to $7.1 \%$ (6.5\% to $7.6 \%$ ) in 2005 , mostly in patients treated by general practitioners and in those with cancer (in 2005, 47\% of sedated patients had cancer $v$ $33 \%$ in 2001). In $83 \%$ of cases sedation was induced by benzodiazepines, and in $94 \%$ patients were sedated for periods of less than one week until death. Nine per cent of those who received continuous deep sedation had previously requested euthanasia but their requests were not granted. Nine per cent of the physicians had consulted a palliative expert.

Conclusions The increased use of continuous deep sedation for patients nearing death in the Netherlands and the limited use of palliative consultation suggests that this practice is increasingly considered as part of regular medical practice.

\section{INTRODUCTION}

Patients nearing death often experience distressing symptoms. ${ }^{12}$ Many patients and physicians are confronted with complex decisions about practices surrounding end of life care that can affect the mode of dying. As an option of last resort, sedating drugs can be used. Such drugs induce a state of decreased consciousness and take away the patient's perception of symptoms. Sedation can be used intermittently or continuously until death, and the depth of the sedation can vary from a lowered state of consciousness to unconsciousness. All these varieties are covered by the term palliative sedation, but sedation to unconsciousness is also referred to as "terminal sedation." Physicians, medical organisations, scientists, ethicists, legal experts, and politicians are debating its use, with discussions focusing on the most extreme use of sedation-that is, continuous deep sedation until death. An important aspect of the debate concern the conditions under which this practice is medically indicated $^{3-8}$ and the way it is performed. ${ }^{8-11}$ On the basis of their expertise and an extensive literature review, an expert group recently recommended that to warrant sedation at the end of life, the patient's condition should be irreversible and advanced, with death expected within at most one to two weeks. ${ }^{8}$ Further recommendations were that benzodiazepines should be the drug of first choice, that hydration should be offered to sedated patients only when the benefit will outweigh the harm, and that advice from palliative care specialists should be sought before sedation.

Another important aspect of the debate is the question of when this practice is ethically acceptable. Is it slow euthanasia or is it a palliative intervention that should be clearly distinguished from euthanasia? ${ }^{12-16}$ When the patient's life expectancy is short when sedation is started, continuous deep sedation presumably has no or only a limited effect on life shortening and is generally not considered to be the moral equivalent of euthanasia. When it is used for patients with a longer life expectancy with the intention to hasten the patient's death at his or her request, however, this practice should be regarded as the moral equivalent of euthanasia.

There is a lack of systematic large scale research on the use of continuous deep sedation because of the methodological, practical, and ethical difficulties related to the vulnerability of patients in their last phase of life. Most studies focus on particular settings and use different definitions. In 2001, a large scale study in six European countries showed that continuous deep sedation was used until death in 2.5-8.5\% of all deaths, among patients with cancer and other diseases, and was provided in hospital as well as outside 
hospital. ${ }^{17}$ For the Netherlands the estimated proportion was 5.6\%. ${ }^{17}$ Another study with a different design and a broader definition estimated that deep sedation until death was used in about $10 \%$ of all deaths in the Netherlands in 2001. ${ }^{18}$

In 2005, as part of a larger study that evaluated the Dutch Euthanasia Act, we repeated the large scale nationwide study in the Netherlands. ${ }^{19}$ The main findings of this study were that the enactment of the Dutch Euthanasia Act was followed by a modest decrease in the rates of euthanasia (from 2.6\% of all deaths in 2001 to $1.7 \%$ of all deaths in 2005) and an increased application of continuous deep sedation until death (from 5.6\% in 2001 to $7.1 \%$ in 2005). The increased use of a method that evokes such controversy, and at the same time represents an indispensable last resort option for unbearably suffering patients, indicates the need for more detailed insight. We compared consecutive data from 2005 with those from 2001 to provide an overview of recent developments in the use of continuous deep sedation in the Netherlands. We focused on the characteristics of the practice of continuous deep sedation until death and the patients who received such sedation.

\section{METHODS}

Study design and data collection

In 2005 , we created a stratified sample of death certificates from the central death registry of Statistics Netherlands to which all deaths are reported..$^{19} \mathrm{We}$ assigned all 43959 deaths that occurred between August and November 2005 to one of five groups, according to the circumstances of death. In group 1 the cause of death precluded any physician assistance in dying (such as a car crash resulting in instant death), and we did not send out a questionnaire. Sampling fractions were larger for the groups in which it was more likely that a physician had assisted in the death. For all sampled cases in which we could not exclude the possibility of physician assistance, we sent the attending physicians a four page questionnaire. The final sample contained half the cases in group 5 (information on the death certificate made an end of life decision likely), $25 \%$ of the cases in group 4 (deaths from cancer

\begin{tabular}{|c|c|c|c|c|}
\hline & \multicolumn{3}{|c|}{ Continuous deep sedation 2005} & \multirow{2}{*}{$\begin{array}{l}\text { Continuous deep } \\
\text { sedation in } \\
\text { conjunction with } \\
\text { ELD, } 2001\end{array}$} \\
\hline & All patients & $\begin{array}{l}\text { Not in conjunction } \\
\text { with ELD }\end{array}$ & $\begin{array}{c}\text { In conjunction with } \\
\text { ELD }\end{array}$ & \\
\hline All physicians & 8.2 & 1.0 & $7.1(6.5$ to 7.6$)$ & $5.6(5.0$ to 6.2$)$ \\
\hline General practitioners & 7.4 & 0.8 & $6.6(5.7$ to 7.6$)$ & $3.9(3.3$ to 4.7$)$ \\
\hline Clinical specialists & 12 & 2.0 & $10.0(8.7$ to 11.5$)$ & 8.4 (7.0 to 10$)$ \\
\hline $\begin{array}{l}\text { Nursing home } \\
\text { physicians }\end{array}$ & 6.4 & 0.5 & $5.9(4.8$ to 7.3$)$ & $7.6(6.3$ to 9.1$)$ \\
\hline
\end{tabular}

$E L D=e n d$ of life decision that potentially hastened death (such as withholding and withdrawing potentially life prolonging treatment).

${ }^{*}$ Concerns all cases of continuous deep sedation in $2005(n=521)$, cases of continuous deep sedation in 2005 not provided in conjunction with end of life decision $(n=67)$, and cases of continuous deep sedation provided in conjunction with end of life decision ( $n=454$ in 2005; $n=336$ in 2001).

tWeighted for sampling fractions, non-response, and random sampling deviations. and deaths that were probably preceded by long term terminal illness), $12.5 \%$ of those in group 3 (nonsudden death from chronic disease), $8.3 \%$ of those in group 2 (sudden death with pre-existing disease), and all cases in group 1 . The 2001 study was similar. ${ }^{20}$ The design of both studies was largely the same, and further details have been described elsewhere. ${ }^{1920}$

\section{Questionnaire}

For all sampled cases in which we could not preclude medical decision making before death, we asked the attending physician whether death had occurred suddenly and unexpectedly. If cases were reported as not sudden, we asked the attending physician to fill in a four page written questionnaire about the medical decision making that had preceded death. Of the 6860 questionnaires sent out, 5342 were completed and returned (78\% response). The response percentage in 2001 was $74 \%(\mathrm{n}=5189)$.

In 2005, the question that pertained to our definition of continuous deep sedation was: "Was the patient continuously and deeply sedated or kept in coma until death?" Follow-up questions were "Which medication was given for sedation?" (midazolam, other benzodiazepine, morphine or a morphine derivative, or other types of medication) and "How long before the patient's death was continuous sedation started?" We asked these questions for all non-sudden deaths. In 2001, physicians were asked "Did the patient receive drugs, such as barbiturates or benzodiazepines, to keep him/her continuously in deep sedation or coma until death?" In 2001, this was asked only for deaths where an end of life decision had (potentially) hastened death, such as intensified alleviation of symptoms with a potential effect on life shortening or the withholding of potentially life prolonging treatments.

The 2005 questionnaire contained additional questions referring to aspects of medical care and treatment before the patient's death and the presence of symptoms, despite possible treatment, during the last 24 hours of life. It also asked whether an expert in palliative care was consulted during the month before death. Physicians were asked to estimate the effect on life shortening, if any, of the decision making before death. We collected data regarding the patient's age, sex, and cause of death from the death certificate.

\section{Analyses}

The percentages reported are weighted to adjust for differences in the sampling fractions and for differences in response rates in relation to the patient's sex, age, marital status, region of residence, and place and cause of death. After adjustment, we extrapolated the percentages to cover a 12 month period to reflect the 136402 deaths in the Netherlands in 2005. Weighting factors were calculated in three steps. Firstly, we took the inverse of the percentage of deaths sampled from each group and multiplied the resulting factor by a second factor that was calculated by dividing the sampled number of deaths by the number of deaths for which we received a questionnaire from the physician 
for each combination of characteristics of patients. The weighting factor that resulted from step 1 and 2 was multiplied by a factor that was calculated in the third step, by dividing the actual number of cases in the population of dead people in 2005 for each combination of characteristics of patients by the number of cases from the first two weighting steps.

We calculated confidence intervals for the main findings. We compared the prevalence of symptoms in patients for whom continuous deep sedation had been started during the last 24 hours of life with the prevalence of symptoms in all patients in whom death was not sudden. We excluded missing values when these comprised less than $5 \%$ of all cases. Statistical analyses were performed with SPSS 11.0 (SPSS, Chicago, IL).

\section{RESULTS}

Table 1 shows the incidence of continuous deep sedation in the Netherlands by specialty of the physician. As reported previously, ${ }^{19}$ of all patients who died in 2005, 8.2\% (95\% confidence interval 7.6\% to $8.9 \%$ ) were continuously and deeply sedated until death. In $7.1 \%(6.5 \%$ to $7.6 \%)$ of deaths, such sedation was provided in conjunction with decisions that potentially hastened death (such as decisions to withhold potentially life prolonging treatments), which is a significant increase compared with the $5.6 \%$ (5.0\% to $6.2 \%)$ in 2001. This increase was significant among general practitioners: $3.9 \%$ (3.3\% to $4.7 \%)$ in $2001 v$ $6.6 \%(5.7 \%$ to $7.6 \%)$ in 2005.Compared with the other specialties, in 2005 the percentage of continuous deep sedation was highest $(10 \%, 8.7 \%$ to $11.5 \%)$ in patients attended by clinical specialists.

Sixty one per cent of patients who received continuous deep sedation were aged $<80$ years, while
$51 \%$ of all deaths in the Netherlands were in those aged $<80$ (table 2). In 2005, $47 \%$ (42\% to $52 \%$ ) of patients who received continuous deep sedation had cancer, compared with $33 \%$ (28\% to 38\%) in 2001. In general practice, the proportions of those with cancer were $72 \%$ in 2005 and $69 \%$ in 2001. Among clinical specialists, continuous deep sedation was also commonly used for patients with cardiovascular diseases (19\%). Nursing home physicians used continuous deep sedation for patients with cardiovascular diseases (24\%) and diseases related to the nervous system $(12 \%)$.

For $47 \%$ of all patients who received continuous deep sedation, the sedation was started in the last 24 hours before death (see table 4). For these patients, 42-55\% were reported as experiencing pain, fatigue, dyspnoea, and unclear consciousness in the last 24 hours of life and $23 \%$ and $21 \%$ had confusion and anxiety (table 3 ). Most patients had more than one symptom, and 74\% experienced symptoms that are common indications for sedation - that is, pain, dyspnoea, confusion, or anxiety. Sedated patients had more symptoms than other patients who did not die suddenly and more often experienced dyspnoea, pain, and anxiety.

Table 4 examines the characteristics of the practice of continuous deep sedation in 2005. No comparable data were available from 2001. In $83 \%$, continuous deep sedation was induced with benzodiazepines often combined with morphine. Such a combination was most often used by clinical specialists. Morphine was used without benzodiazepines in 15\%. Nursing home physicians and general practitioners reported using benzodiazepines relatively often $(89 \%$ and $87 \%$, respectively), whereas clinical specialists were more likely to administer morphine for sedation (19\% of cases). Palliative consultation in the month before death was quite rare $(9 \%)$, and most often sought by

Table 2 | Characteristics of patients who received continuous deep sedation and of all deaths in the Netherlands . Figures are percentages $\dagger$ of patients with $95 \%$ confidence intervals where applicable

\begin{tabular}{|c|c|c|c|c|c|c|c|c|c|c|}
\hline & \multirow{2}{*}{\multicolumn{2}{|c|}{ All deaths }} & \multicolumn{6}{|c|}{ Patients attended by } & \multirow{2}{*}{\multicolumn{2}{|c|}{ All physicians }} \\
\hline & & & \multicolumn{2}{|c|}{ General practitioners } & \multicolumn{2}{|c|}{ Clinical specialists } & \multicolumn{2}{|c|}{$\begin{array}{c}\text { Nursing } \\
\text { home } \\
\text { physicians }\end{array}$} & & \\
\hline & 2005 & 2001 & 2005 & 2001 & 2005 & 2001 & 2005 & 2001 & 2005 & 2001 \\
\hline \multicolumn{11}{|l|}{ Age (years): } \\
\hline $0-64$ & 19 & 20 & 28 & 34 & 32 & 27 & 11 & 7 & 26 (23 to 30$)$ & 23 (19 to 27 ) \\
\hline $65-79$ & 32 & 35 & 37 & 40 & 38 & 52 & 28 & 46 & 35 (31 to 40$)$ & $47(42$ to 52$)$ \\
\hline$>80$ & 48 & 46 & 35 & 26 & 30 & 21 & 60 & 47 & 39 (34 to 44$)$ & 30 (25 to 35$)$ \\
\hline \multicolumn{11}{|l|}{ Sex: } \\
\hline Male & 49 & 49 & 50 & 48 & 57 & 60 & 44 & 35 & 51 (46 to 56$)$ & 50 (45 to 55$)$ \\
\hline Female & 51 & 51 & 50 & 52 & 43 & 40 & 56 & 65 & $49(44$ to 54$)$ & $50(45$ to 55$)$ \\
\hline \multicolumn{11}{|l|}{ Cause of death $\ddagger$ : } \\
\hline Cancer & 29 & 27 & 72 & 69 & 33 & 19 & 34 & 24 & $47(42$ to 52$)$ & 33 (28 to 38$)$ \\
\hline Cardiovascular disease & 32 & 25 & 11 & 6 & 19 & 20 & 24 & 8 & 17 (13 to 22$)$ & 13 (9 to 18$)$ \\
\hline Pulmonary disease & 10 & 10 & 3 & 8 & 8 & 7 & 2 & 15 & $6(4$ to 10$)$ & 10 (7 to 14$)$ \\
\hline Nervous system disease & 3 & 10 & 1 & 3 & 2 & 8 & 12 & 14 & $4(2$ to 7$)$ & $8(6$ to 12$)$ \\
\hline Other/unknown & 26 & 27 & 12 & 14 & 38 & 47 & 27 & 39 & 26 (22 to 31$)$ & 36 (31 to 42$)$ \\
\hline
\end{tabular}

${ }^{*}$ Cases of continuous deep sedation provided in conjunction with end of life decision ( $n=454$ in 2005, $n=336$ in 2001).

†Weighted for sampling fractions, non-response, and random sampling deviations.

$\ddagger$ Cerebrovascular disease included in diseases of nervous system in 2001 and in cardiovascular diseases in 2005. 
Table $3 \mid$ Signs or symptoms ${ }^{\star}$ in last 24 hours of life for deaths preceded by continuous deep sedation and for all other non-sudden deaths in 2005. Figures are percentages $\dagger$ of patients with $95 \%$ confidence intervals

Continuous deep sedation $\ddagger$ All non-sudden deaths§

Symptoms:

\begin{tabular}{lcc}
\hline Fatigue & $55(49$ to 61$)$ & 48 (46 to 50$)$ \\
\hline Dyspnoea & $48(42$ to 54$)$ & 31 (29 to 33$)$ \\
\hline Unclear consciousness & $47(41$ to 53$)$ & 40 (38 to 42$)$ \\
\hline Pain & $42(36$ to 48$)$ & $19(18$ to 20$)$ \\
\hline Confusion & $23(18$ to 29$)$ & 18 (17 to 19$)$ \\
\hline Anxiety & $21(16$ to 27$)$ & $9(8$ to 10$)$ \\
\hline Vomiting & $5(3$ to 8$)$ & $4(4$ to 5$)$ \\
\hline Depressive symptoms & $3(1$ to 6$)$ & $3(3$ to 4$)$ \\
\hline
\end{tabular}

No of symptoms:

\begin{tabular}{lll}
0 & 7 & 17 \\
\hline 1 & 22 & 32 \\
\hline 2 & 27 & 27 \\
\hline 3 & 43 & 24 \\
\hline
\end{tabular}

* Score 4 or 5 on a scale of 1 (symptom not present) to 5 (symptom strongly present despite treatment).

†Weighted for sampling fractions, non-response, and random sampling deviations

fln patients in whom sedation was started in 0-24 hours before death $(n=238)$. Symptoms missing for $6-16 \%$ of patients.

§Excludes cases of continuous deep sedation ( $n=3334)$. Symptoms were missing for $7-14 \%$ of patients.

general practitioners (20\% of cases). Palliative consultation was positively related to the use of benzodiazepines (whether or not combined with other drugs) $(\mathrm{P}<0.01)$. For almost all of the patients for whom sedation was used, general practitioners and nursing home physicians withheld artificial nutrition and hydration, whereas this was true for $30 \%$ of the patients attended by clinical specialists. In two thirds, physicians estimated that the effect of the decision making before death on shortening life was 24 hours or less. In $6 \%$ of the cases, patients were sedated for more than one week ( $11 \%$ among clinical specialists). In $9 \%$ of the cases, the decision to use continuous deep sedation was preceded by an explicit request from the patient to end his or her life by means of euthanasia or assisted suicide, which was not granted. General practitioners reported this more often $(16 \%)$ than clinical specialists $(4 \%)$ and nursing home physicians $(9 \%)$. The most frequently cited reason why the physician had not granted the patient's request was that the time frame to complete the euthanasia procedure was too short-for example, because of the obligation to consult another physician $(40 \%, n=24)$. Other reasons mentioned were that the suffering of the patient was not considered to be unbearable $(10 \%, \mathrm{n}=4)$ and that the patient had withdrawn the request for euthanasia $(9 \%, n=6)$.

\section{DISCUSSION}

In the Netherlands the increase in the use of continuous deep sedation for patients nearing death mostly occurred among general practitioners and clinical specialists, especially for patients with cancer. Patients for whom sedation was started in the last 24 hours of their life more often had dyspnoea, pain, and anxiety than other patients whose death was not sudden. In about four out of every five patients sedation was induced with benzodiazepines. Clinical specialists were more likely than general practitioners and nursing home physicians to use other drugs, mostly morphine. Physicians rarely sought palliative consultation, although this happened more often among general practitioners. In about one in every 10 patients, the use of continuous deep sedation was preceded by a request for euthanasia or assisted suicide that was not granted.

\section{Strengths and weaknesses of the study}

The large random samples of death certificates and high response rates in both years ensure generalisability to all deaths in the Netherlands. That the study design was similar in both years, authoritative medical bodies endorsed the study, and anonymity of patients and physicians was guaranteed further strengthen the validity and reliability of our results. In the 2005 questionnaire, there were small adjustments in the wording of the key questions regarding the use of continuous deep sedation. Because we did not change the key elements of our definition (continuous and deep sedation or coma until death) we believe this did not affect our results.

Strengths and weaknesses in relation to other studies To minimise possible differences in the perception of sedation across the respondents we provided them with a descriptive definition of the practice (continuous deep sedation until death). Most other studies use terms such as palliative or terminal sedation, which can have varying connotations and implications for normal practice.

In 2005 continuous deep sedation until death was used in $8.2 \%$ of all deaths. The proportion of continuous deep sedation was highest $(10 \%)$ among patients attended by clinical specialists compared with the other specialties. This is in line with a study performed in six European countries in 2001, where the highest rates of continuous deep sedation were also found among patients who died in hospitals compared with elsewhere (2.9-13.2\%), except for Italy, where sedation rates were higher outside the hospital. ${ }^{17}$ Furthermore, our findings show an increase in the use of continuous deep sedation until death in the studied period, especially among general practitioners. This agrees with results of another Dutch longitudinal study. ${ }^{21}$

While half of the sedated patients in our study had cancer, continuous deep sedation was also used for patients with cardiovascular diseases, pulmonary diseases, and diseases of the nervous system, similar to the 2001 European study. ${ }^{17}$ Most other studies report the use of continuous deep sedation only for patients with cancer, probably because they were restricted to specific settings such as palliative care units. ${ }^{22-25}$ Furthermore, in the last 24 hours of life, many patients for whom sedation was started in this period experienced dyspnoea, pain, and fatigue. Anxiety and confusion were reported for one in five patients. Symptoms reported by other studies were not restricted to the last 24 hours and focused on indications rather than on symptoms. In those studies, delirium or terminal restlessness, dyspnoea, and pain 
are frequently mentioned indications for sedation, ${ }^{1822-242627}$ which seems to agree with our findings.

We found that $15 \%$ of patients were sedated with morphine as a single therapy. Two other nationwide Dutch studies conducted in 2001 and 2005 found somewhat higher levels of use $\left(36 \%{ }^{18}\right.$ and $28 \%,{ }^{10}$ respectively). Similar to our findings, both studies showed that clinical specialists were more likely than general practitioners and nursing home physicians to use morphine for this purpose. ${ }^{10}{ }^{18}$ Most patients (94\%) were sedated for less than one week, which is also comparable with results of other studies..$^{22-2428}$

\section{Interpretation}

There could be several explanations for the increase in the use of continuous deep sedation until death seen between 2001 and 2005. Physicians and medical authorities have paid more attention to continuous deep sedation and knowledge has increased. From 2002 on, for example, regional cancer centres have published guidelines to improve its application, and in 2005 (after our data collection) a nationwide guideline was released in the Netherlands. ${ }^{29}$ The practice of continuous deep sedation until death also recently received a lot of attention in the media. These developments could have led to an increased interest in its use among physicians as well as patients and their relatives.
Continuous deep sedation has possibly increasingly been used as a relevant alternative to euthanasia. The use of euthanasia had decreased from $2.6 \%$ of all deaths in 2001 to $1.7 \%$ of all deaths in 2005 (a decrease of 1200 cases), ${ }^{19}$ while continuous deep sedation increased by 1800 cases from $5.6 \%$ to $7.1 \%$. This increase took place mostly in the subgroups in which euthanasia is most common: patients attended by general practitioners and those with cancer. In $9 \%$ of the patients, the use of continuous deep sedation was preceded by a request for euthanasia that was not granted. Although euthanasia and continuous deep sedation generally address different clinical problems, ${ }^{16}$ this suggests that substitution might be possible in some situations. This is in line with the finding that many Dutch physicians have been found to consider high quality end of life care as an alternative to euthanasia. ${ }^{20} \mathrm{We}$ do not know whether such substitution is always in accordance with the patient's wishes and with legal and professional guidelines. As long as the life expectancy of the patient is short at the time that sedation is started, continuous deep sedation presumably has no or only a limited effect on life shortening. In such cases, continuous deep sedation might be considered as normal medical practice, which does not require special judicial supervision such as with euthanasia. For patients with a longer life expectancy, there is a risk that labelling the decision as continuous deep sedation instead of ending of life might serve as a way to evade the procedural

\begin{tabular}{|c|c|c|c|c|}
\hline & General practitioners & Clinical specialists & Nursing home physicians & All physicians \\
\hline \multicolumn{5}{|l|}{ Drugs administered: } \\
\hline Benzodiazepines & 42 & 13 & 44 & 30 \\
\hline Benzodiazepines and morphine & 44 & 60 & 43 & 51 \\
\hline $\begin{array}{l}\text { Benzodiazepines and other } \\
\text { drugs }\end{array}$ & 1 & 3 & 2 & 2 \\
\hline Morphine & 11 & 16 & 10 & 13 \\
\hline Morphine and other drugs & 2 & 3 & 0 & 2 \\
\hline Other drugs & 0 & 5 & 0 & 2 \\
\hline $\begin{array}{l}\text { Palliative consultation in last } \\
\text { month before death }\end{array}$ & 20 & 2 & 5 & 9 \\
\hline $\begin{array}{l}\text { Artificial nutrition or hydration } \\
\text { withheld during sedation }\end{array}$ & 95 & 30 & 98 & 66 \\
\hline \multicolumn{5}{|l|}{ Duration of continuous deep sedation: } \\
\hline $0-24$ hours & 43 & 50 & 42 & 47 \\
\hline $1-7$ days & 52 & 38 & 58 & 47 \\
\hline 1-2 weeks & 3 & 7 & 0 & 4 \\
\hline >2 weeks & 2 & 4 & 0 & 2 \\
\hline \multicolumn{5}{|l|}{ Estimated shortening of lifeł: } \\
\hline None or $<24$ hours & 57 & 73 & 64 & 65 \\
\hline $1-7$ days & 27 & 17 & 18 & 20 \\
\hline 1-4 week & 6 & 3 & 1 & 4 \\
\hline$>1$ month & 0 & 3 & 4 & 2 \\
\hline Unknown/missing & 9 & 5 & 13 & 9 \\
\hline $\begin{array}{l}\text { Preceded by request for } \\
\text { euthanasia, not granted }\end{array}$ & 16 & 4 & 9 & 9 \\
\hline
\end{tabular}




\section{WHAT IS ALREADY KNOWN ON THIS TOPIC}

Continuous deep sedation until death is sometimes used in severely suffering patients with refractory symptoms; in the Netherlands use increased from $5.6 \%$ of all deaths in 2001 to $7.1 \%$ in 2005

Guidelines recommend the use of benzodiazepines for deep sedation, while morphine is usually rejected as a single therapy to attain sedation because of its unpredictable sedative and side effects

Continuous deep sedation and euthanasia are generally used in different clinical situations

\section{WHAT THIS STUDY ADDS}

The increase in the use of continuous deep sedation until death in the Netherlands was mostly because of an increase in use by general practitioners, especially for patients with cancer

Of the physicians who used continuous deep sedation, 15\% used morphine and no benzodiazepines and $91 \%$ did not consult a palliative care expert

The use of euthanasia decreased while the use of continuous deep sedation increased, especially in subgroups in which euthanasia is most common; and $9 \%$ of patients who received continuous deep sedation had previously asked for euthanasia but their requests were not granted for the use of this practice. Lastly, rendering patients unconscious until death is a far reaching intervention that has an important impact on the dying process for both the patient and the relatives. Whether and how such sedation contributes to a dignified death deserves further study.

We thank the thousands of physicians who provided the study data, the assistants at Statistics Netherlands for their skilful help in collecting the data, and the members of the steering committee, the Royal Medical Association, and the chief inspector for health care for their support of the study.

Contributors: JvD, BOP, PvdM, and AvdH designed the study. JR and HB analysed the data. JR wrote the paper, which was critically read by all the authors. AvdH is guarantor.

Funding: Dutch Ministry of Health.

Competing interests: None declared.

Ethical approval: Not needed.

Provenance and peer review: Not commissioned; externally peer reviewed.

1 Vainio A, Auvinen A. Prevalence of symptoms among patients with advanced cancer: an international collaborative study. Symptom prevalence group. J Pain Symptom Manage 1996;12:3-10.

2 Klinkenberg M, Willems DL, van der Wal G, Deeg DJ. Symptom burden in the last week of life. J Pain Symptom Manage 2004;27:5-13.

requirements for euthanasia. Our study shows, however, that the estimated effect on life shortening was, in most cases, limited. Future studies about medical practices at the end of life should evaluate this in more detail.

Around 15\% of the physicians used morphine without a benzodiazepine to attain sedation. Most guidelines at present recommend the use of benzodiazepines for deep sedation, while morphine is usually rejected as a single therapy to attain sedation because of its unpredictable sedative and side effects. A previous Dutch study suggested that the choice of drugs for continuous deep sedation is associated with the use of guidelines and being a palliative expert or consultation of a palliative care expert. ${ }^{10}$ Our study confirmed the relation between consultation and adequate drug use. Such consultation, however, occurred in only a small number of the cases in our study, maybe because most of the physicians consider the application of continuous deep sedation as normal medical practice that is part of their own expertise or because they were not well informed about it or did not have access to palliative consultation. Increased use of palliative consultation might improve the clinical performance of continuous deep sedation.

\section{Unanswered questions and future research}

Future research should focus on the underlying reasons for the use of continuous deep sedation. The perspectives of physicians as well as the wishes of patients and relatives should be taken into account. Specific attention should be directed at the substitution of euthanasia by continuous deep sedation, especially in general practice because euthanasia in the Netherlands usually takes place at home. Research should include a longitudinal timeframe to investigate how the practice of continuous deep sedation will further develop. This should be done nationally and internationally to allow comparisons and increase understanding of the reasons
3 Gillick MR. Terminal sedation: an acceptable exit strategy? Ann Intern Med 2004;141:236-7.

4 Quill TE, Lee BC, Nunn S. Palliative treatments of last resort: choosing the least harmful alternative. University of Pennsylvania Center for Bioethics Assisted Suicide Consensus Panel. Ann Intern Med 2000;132:488-93.

5 Quill TE, Lo B, Brock DW. Palliative options of last resort: a comparison of voluntarily stopping eating and drinking, terminal sedation, physician-assisted suicide, and voluntary active euthanasia. JAMA 1997;278:2099-104.

6 Rousseau P. Existential suffering and palliative sedation: a brief commentary with a proposal for clinical guidelines. Am J Hosp Palliat Care 2001;18:151-3.

7 Morita T, Tei Y, Inoue S. Ethical validity of palliative sedation therapy.J Pain Symptom Manage 2003;25:103-5.

8 De Graeff A, Dean M. Palliative sedation therapy in the last weeks of life: a literature review and recommendations for standards. J Palliat Med 2007;10:67-85.

9 Gahan C, Hannibal M. Doing qualitative research using QSR Nudist. London: Sage, 1998.

10 Hasselaar JG, Reuzel RP, Verhagen SC, de GraeffA, Vissers KC, Crul BJ. Improving prescription in palliative sedation: compliance with Dutch guidelines. Arch Intern Med 2007;167:1166-71.

11 Goncalves JA. Sedation and expertise in palliative care. J Clin Oncol 2006;24:e44-5.

12 Billings IA, Block SD. Slow euthanasia. I Palliat Care 1996;12:21-30.

13 Billings JA, Block SD. Opportunity to present our observations and opinions on slow euthanasia. J Palliat Care 1997;13:55-6.

14 Van Delden JJ. Terminal sedation: source of a restless ethical debate.J Med Ethics 2007;33:187-8.

15 Tännsjö T. A substitute for euthanasia? In: Tännsjö T, ed. Terminal sedation: euthanasia in disguise? Dordrecht: Kluwer Academic Publishers, 2004.

16 Rietjens JA, van Delden JJ, van der Heide A, Vrakking AM, Onwuteaka-Philipsen BD, van der Maas PJ, et al. Terminal sedation and euthanasia: a comparison of clinical practices. Arch Intern Med 2006;166:749-53.

17 Miccinesi G, Rietjens JA, Deliens L, Paci E, Bosshard G, Nilstun T, et al. Continuous deep sedation: physicians' experiences in six European countries. J Pain Symptom Manage 2006;31:122-9.

18 Rietjens JA, van der Heide A, Vrakking AM, Onwuteaka-Philipsen BD, van der Maas PJ, van der Wal G. Physician reports of terminal sedation without hydration or nutrition for patients nearing death in the Netherlands. Ann Intern Med 2004;141:178-85.

19 Van der Heide A, Onwuteaka-Philipsen BD, Rurup ML, Buiting HM, van Delden JJ, Hanssen-de Wolf JE, et al. End-of-life practices in the Netherlands under the Euthanasia Act. N Engl J Med 2007;356:1957-65.

20 Onwuteaka-Philipsen BD, van der Heide A, Koper D, Keij-Deerenberg Rietjens JA, Rurup ML, et al. Euthanasia and other end-of-life decisions in the Netherlands in 1990,1995, and 2001. Lancet 2003;362:395-9.

21 Stichting Farmaceutische Kengetallen. De richtlijn palliatieve sedatie. [Palliative sedation directive.] Pharm Weekbl 2006;141(3):81.

22 Muller-Busch HC, Andres I, Jehser T. Sedation in palliative care-a critical analysis of 7 years experience. BMC Palliat Care 2003;2:2. 
23 Fainsinger RL, Waller A, Bercovici M, Bengtson K, Landman W, Hosking $\mathrm{M}$, et al. A multicentre international study of sedation for uncontrolled symptoms in terminally ill patients. Palliat Med 2000;14:257-65.

24 Chiu TY, Hu WY, Lue BH, Cheng SY, Chen CY. Sedation for refractory symptoms of terminal cancer patients in Taiwan. J Pain Symptom Manage 2001;21:467-72.

25 Stone P, Phillips C, Spruyt O, Waight C. A comparison of the use of sedatives in a hospital support team and in a hospice. Palliat Med 1997;11:140-4.

26 Sykes N, Thorns A. The use of opioids and sedatives at the end of life. Lancet Oncol 2003;4:312-8.
27 Kohara H, Ueoka H, Takeyama H, Murakami T, Morita T. Sedation for terminally ill patients with cancer with uncontrollable physical distress. J Palliat Med 2005;8:20-5.

28 Rietjens JA, van der Heide A, Onwuteaka-Philipsen BD, van der Maas PJ, van derWal G. A comparison of attitudes towards end-of-life decisions: survey among the Dutch general public and physicians. Soc Sci Med 2005;61:1723-32.

29 Verkerk M, van WijlickE, Legemaate J, de GraeffA. A national guideline for palliative sedation in the Netherlands. J Pain Symptom Manage 2007;34:666-70.

Accepted: 15 February 2008 